\title{
224Gb/s Single Carrier Doubly Differential 2ASK-8PSK System without Carrier Recovery
}

Tingting Zhang, Christian Sanchez, Mohammad Al-Khateeb, Ian Phillips, Andrew Ellis

Aston Institute of Photonic Technologies (AIPT), Aston University, Birmingham, B4 7ET, UK

Authore-mail address: zhangt16@aston.ac.uk

\begin{abstract}
A carrier-recovery-free 224Gb/s dual-polarization doubly differential 16-point constellation (2ASK-8PSK) is experimentally demonstrated for the first time. Utilizing 10-symbol decision feedback in the $2^{\text {nd }}$ differential stage, the penalty with respect to conventional $16 Q A M$ is only 4.8dB.

OCIS codes: (060.1660) Coherent communications; (060.2330) Fiber optics communications; (060.4080) Modulation
\end{abstract}

\section{Introduction}

With the emergence of price-sensitive applications such as mega-data centers, which are currently supported by $100 \mathrm{~Gb} / \mathrm{s}$ transceivers and shortly by $400 \mathrm{~Gb} / \mathrm{s}$ products, attention is focused on increasing the data rate $(>100 \mathrm{~Gb} / \mathrm{s})$ per wavelength to reduce overall complexity and cost. Many schemes employing high-order modulation format [1], high symbol rate [2], or multi-carrier techniques [3] have been reported. These solutions typically use a digital coherent receiver architecture with powerful digital signal processing (DSP) utilized to compensate for transmission impairments and bandwidth limitation. Despite increased optical system complexity, coherent solutions fundamentally achieve higher capacity due to the ready access to all four field dimensions and significantly improved noise performance. However, challenges remain to reduce DSP complexity and improve energy efficiency for higher order modulation formats. In [4], a 200Gb/s doubly differential (DD) encoded QPSK system with simplified receiver-side DSP was reported. Although $2 \mathrm{GHz}$ frequency offset tolerance with negligible power penalty was numerically demonstrated, the information spectral density was limited due to the use of QPSK and guard bands, and the receiver sensitivity penalty was degraded by 7.1dB compared to a conventional QPSK system.

In this paper, we propose and experimentally demonstrate a single carrier $224 \mathrm{~Gb} / \mathrm{s}$ carrier-recovery-free system using DD detection of a 16-point constellation, two-amplitude/eight-phase shift keyed (2ASK-8PSK) for the first time. Experimental results show 8.3dB (theoretically 7.17dB) penalty between conventional DDQPSK and QPSK, which is reduced by $4.4 \mathrm{~dB}$ with the help of multi-symbol doubly differential (MSDD) decoding. By resorting to this technique, carrier-recovery-free DD 2ASK-8PSK system only suffers a $4.8 \mathrm{~dB}$ penalty when compared with a conventional 16QAM system with full carrier recovery.

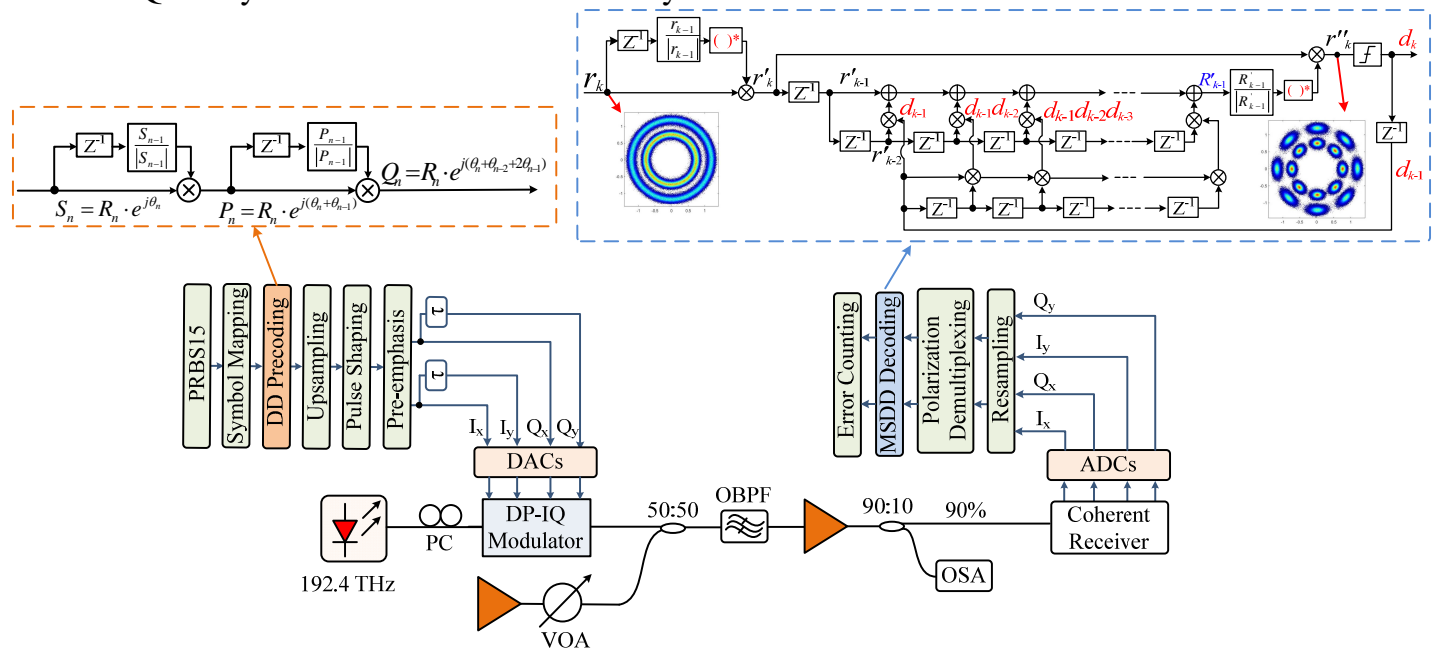

Fig. 1 Experimental setup for 224Gb/s DD 2ASK-8PSK system. DD precoding and decoding are shown in the orange and blue dashed boxes.

\section{Experimental setup}

The experimental setup for the proposed dual-polarization DD 2ASK-8PSK system is depicted in Fig. 1. A 28Gbaud/s 2ASK-8PSK signal with normalized radii of 0.725 and 1.214 was firstly generated in Matlab with $2^{15}-1$ pseudo random binary sequences before DD precoding (shown in orange dashed box of Fig. 1). After resampling to 2 Samples/symbol, the DD encoded signal was pulse shaped employing a square root raised cosine filter with roll- 
off factor of 0.3. The signal for the Y polarization was generated by introducing a decorrelation length of 8192 samples compared to the $\mathrm{X}$ polarization signal, both of which were then loaded to the memories of 4 digital to analogue converters (DACs) running at 56GS/s. The output signals of the four DACs were fed to a dual-polarization (DP) IQ modulator before coupling with amplified spontaneous emission noise from erbium-doped fiber amplifier with a variable optical attenuator inserted to change the optical signal to noise ratio (OSNR). A wavelength selective switch was utilized as an optical bandpass filter (OBPF) with bandwidth of 250GHz. The filtered signal was amplified again and input to a coherent receiver (Tektronix, OM4245) with a free-running local oscillator. An optical spectrum analyzer (Finisar WaveAnalyzer 1500s) with resolution bandwidth of $150 \mathrm{MHz}$ was used to measure the OSNR. The data was captured at 100GS/s and firstly resampled to 2 Samples/symbol and then imbalance compensated and polarization de-multiplexed using eight 25-tap adaptive finite impulse response filters with real-valued weights optimized through a radius-directed constant magnitude algorithm. Instead of implementing carrier recovery, MSDD decoding with 10 -symbol decision feedback in the $2^{\text {nd }}$ differential stage (shown in blue dashed box of Fig. 1) was adopted in our system. After decoding, the symbol error rate (SER) was calculated for each trace with at least 65000 symbols per calculation, and the average SER together with standard deviation was recorded from ten separate measurements.

\section{Results and discussion}

We investigated the back-to-back transmission performance for different modulation formats in terms of OSNR at 0.1-nm reference bandwidth. The OSNR was measured using the spectral integration method at a high-resolution bandwidth (150 MHz) and then converted to a 0.1-nm reference bandwidth. Fig. 3 (a) shows that the implementation penalty in 224Gb/s DP-16QAM system is about $3.2 \mathrm{~dB}$ away from the theory (black dashed line). We believe that this might be due to the clock offset between the transponders. Utilizing conventional DD decoding, the required OSNR at SER of $4 \times 10^{-3}$ for DDQPSK was degraded by $8.3 \mathrm{~dB}$ (theoretically $7.17 \mathrm{~dB}$ ), with respect to QPSK with carrier recovery. The penalty was reduced to $3.9 \mathrm{~dB}$ using MSDD decoding. With the same technique, $4.8 \mathrm{~dB}$ penalty is observed when comparing the performance of MSDD 2ASK-8PSK (Fig. 2 (b)), and 16QAM with full carrier recovery. Without MSDD decoding, the DD 2ASK-8PSK was not decodable at the OSNR levels reported here.
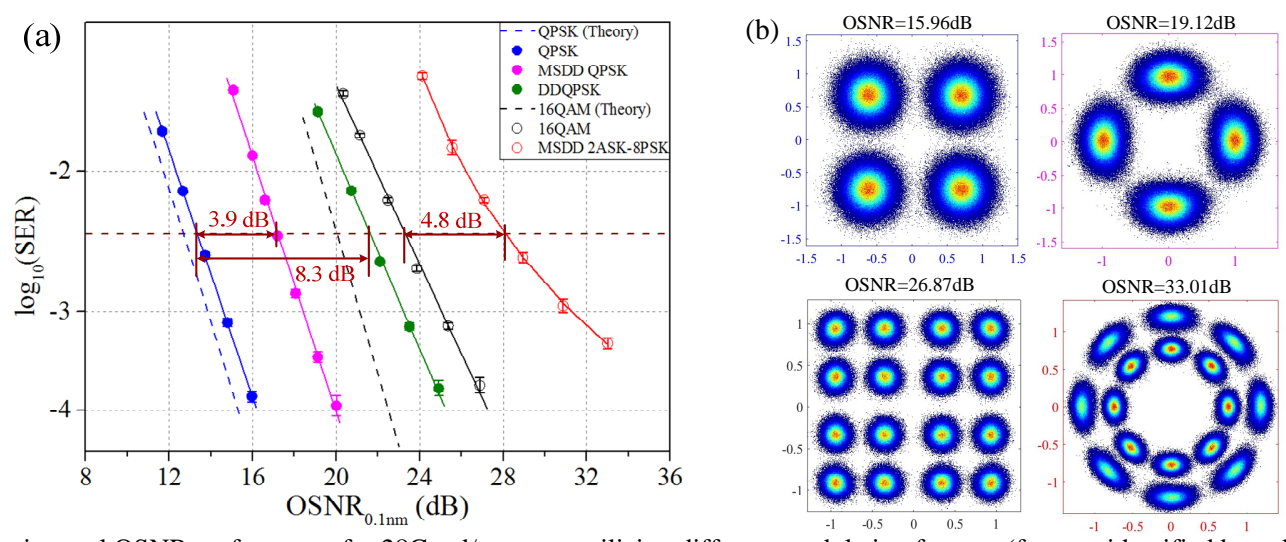

Fig. 2 (a) Experimental OSNR performance for 28Gaud/s system utilizing different modulation formats (formats identified by color, dashed lines for theory). (b) Constellations for QPSK, MSDD QPSK, 16QAM and MSDD 2ASK-8PSK at varied OSNR.

\section{Conclusion and acknowledgements}

We have experimentally demonstrated single carrier data rate of 224Gb/s using DD 2ASK-8PSK modulation format without carrier recovery. We believe this to be the first report of DD decoding of a 16-point constellation. The required OSNR at SER of $4 \times 10^{-3}$ was only degraded by $4.8 \mathrm{~dB}$ compared to 16QAM with full carrier recovery.

This work was partially supported by the China Scholarship Council (CSC), Marie Currie Fellowship (INVENTION), EPSRC projects (PEACE, UPON, and SPFS) and the Royal Society (Grant numbers: 659950, EP/L000091/1, EP/M005283/1, EP/L00044X/1 respectively).

\section{References}

[1] H. Chien, and J. Yu, “On single-carrier 400G line side optics using PM-256QAM,” in Proc. ECOC, 2016.

[2] P. J. Winzer, A. H. Gnauck, S. Chandrasekhar, S. Draving, J. Evangelista, and B. Zhu, "Generation and 1,200-km transmission of 448-Gb/s ETDM 56-Gbaud PDM 16-QAM using a single I/Q modulator," in Proc. ECOC, 2010.

[3] X. Liu, S. Chandrasekhar, B. Zhu, P. J. Winzer, A. H. Gnauck, and D. W. Peckham., "448-Gb/s Reduced-Guard-Interval CO-OFDM Transmission Over 2000 km of Ultra-Large-Area Fiber and Five 80-GHz-Grid ROADMs,” J. Lightwave Technol. 29, 483-490 (2011).

[4] T. T. Zhang, C. Sanchez, I. Phillips, S. Sygletos, A. Ellis, "200-Gb/s Polarization Multiplexed Doubly Differential QPSK Signal Transmission over 80-km SSMF Using Tandem SSB without Optical Amplification,” in Proc. ECOC, P1.SC4.69 (2017). 